\title{
Biofilms at the Mineral-Microbe Interface; Development of Microbial Community Structure and Persistent Soil Organic Matter.
}

\author{
MARJORIE SCHULZ ${ }^{1}$, MARY-CATHRINE LEEWIS ${ }^{2}$, AND
} KRISTEN MANIES ${ }^{3}$

${ }^{1}$ USGS, Menlo Park CA; mschulz@usgs.gov

${ }^{2}$ USGS, Menlo Park CA; mcleewis@usgs.gov

${ }^{3}$ USGS, Menlo Park CA; kmanies@usgs.gov

We do not understand all the reasons soil organic carbon (SOC) endures, although we do know that persistent SOC is found in soil aggregates and associated with short range order (SRO) minerals such as Fe-oxyhydroxides and nanocrystalline clays. There is also data $(\mathrm{C}$ isotopes and $\mathrm{C} / \mathrm{N}$ ratios) to support the theory that this persistent $\mathrm{SOC}$ is microbially processed. However, we do not yet understand the processes occurring at the microbe-mineral interface which create niches for SOC to endure. To examine the role of microbes in the creation of SOC we studied microbial community colonization of fresh mineral surfaces using sterilized minerals (apatite, plagioclase feldspar, K-feldspar, hornblende, and quartz) in nylon mesh bags which were placed in a soil near Santa Cruz, CA. Bags were retrieved after 15 and 24 months. We used amplicon-based sequence analysis (16S rRNA gene and ITS region) to characterize microbial community assemblages on the minerals containing different nutrient elements. After 15 months bacterial and archaeal communities in the mesh bags were distinct from the bulk soil. However, there were no measurable community differences in the measured microbial populations between the minerals. After 24 months, microbial populations furthered this trend of community structure, indistinct based on mineral type. The was no measurable difference in mineral and soil fungal communities.

We used optical and scanning electron microscopy (SEM) to study microbes on the mineral surfaces. We quantified, using ImageJ software, the biofilm coverage on a subset of minerals after 24 months; biofilms covered 2.87 to $9.27 \%$ of mineral surfaces in 50 um mesh bags and 5.62 to $15.81 \%$ in 250 um mesh bags. SEM images established that SOCmineral interfaces are mostly microbial biofilms and their remnants, highlighting the importance of biofilm production in generating SOM. SEM images also reveal that microbial biofilms weather primary mineral grains, and that biologic precipitation of SRO minerals occurs within biofilms, likely co-precipitating with organic compounds thus, trapping SOC from degradation. 